\title{
Context effects in visual pattern recognition by pigeons
}

\author{
FRANCISCO J. DONIS \\ Central Connecticut State University, New Britain, Connecticut \\ ERIC G. HEINEMANN \\ Brooklyn College, City University of New York, New York \\ and \\ SHEILA CHASE \\ Hunter College, City University of New York, New York
}

\begin{abstract}
In the experiments described in this paper we examined the effects of contextual stimuli on pigeons' recognition of visual patterns. Experiment 1 showed a context-superiority effect. Specifically, two target forms that were identical except for location in the visual field were not discriminated when presented alone, but the compounds formed when each of these targets was placed between a nearby pair of flanking stimuli were readily discriminated. The size of the contextsuperiority effect decreased with increasing target-flanker separation. In Experiments 2 and 3 the two targets differed in form rather than spatial location and were readily discriminated in the absence of flankers. Under these circumstances, adding an identical pair of flankers to each target resulted in a context-inferiority effect; that is, the two target-plus-flankers compounds were less readily discriminated than the targets alone. The size of the context-inferiority effect decreased with increasing target-flanker separation. The observed effects of context are predictable from the Heinemann-Chase (1990) model of pattern recognition.
\end{abstract}

Numerous experiments have shown that discrimination between two forms-targets-is affected if each of the targets is accompanied by an identical, and therefore uninformative set of additional forms-contexts (Pomerantz, Sager, \& Stoever, 1977; Weisstein \& Harris, 1974). The effect of the contexts is evaluated by removing them from or adding them to the target forms under study. In accordance with recent usage (Enns \& Prinzmetal, 1984), we shall refer to an improvement in discrimination between forms resulting from the addition of context elements as a context-superiority effect and impairment of discrimination as a result of the same operation as a contextinferiority effect.

Investigators who have studied this phenomenon with human observers have found both context-superiority effects and context-inferiority effects (Enns \& Prinzmetal, 1984; Pomerantz et al., 1977). Which of these effects will be produced by the presence of the common elements seems to depend on the specific configurations that are

\footnotetext{
The research reported here is part of a doctoral dissertation submitted by the first author to the City University of New York in 1991. It was supported by NIMH Grant R01 MH40712 to E.G.H. and S.C., and by PSC-CUNY Grant 669211 to E.G.H. We wish to thank the editor of this article, L. E. Krueger, as well as three anonymous reviewers, for many helpful comments and suggestions on an earlier version of the manuscript. Correspondence concerning this article should be addressed to F. J. Donis, Department of Psychology, Central Connecticut State University, 1615 Stanley Street, New Britain, CT 06050 (e-mail: donis@ccsu.cstateu.edu).
}

studied (Pomerantz et al., 1977), but a rule for predicting the direction of the effects solely on the basis of the stimulus forms has not been found.

In most experiments the contexts themselves have been uninformative, in the sense that their forms were not correlated with the target forms and therefore could not be used to identify the targets. However, much research dealing with context-superiority effects has dealt with the possible effects of correlations between the internal representations of the targets alone and targets in context. For example, Enns and Prinzmetal (1984) experimented with the much-studied stimulus situation in which the target is a straight line tilted $45^{\circ}$ in a clockwise or counterclockwise direction and the context consists of two straight lines in the form of an $\mathbf{L}$. This situation yields a contextsuperiority effect often referred to as an object-line effect.

Enns and Prinzmetal (1984) noted that the compound stimuli formed by combining each target with the Lshaped context had the form of an arrow and a closed triangle, respectively, and that these perceived forms were perfectly correlated with the orientations of the target lines. They proposed that the context-superiority effect found under these conditions reflects a redundancy gainpossible because the compound stimuli can be identified by the perceived orientations of the target lines and by the perceived forms of the compound stimuli (arrow or triangle). This proposal was supported by the results of experiments in which they systematically varied the size of the correlation between the target-orientation and the 
arrow-triangle dimensions and found that the magnitude of the context-superiority effect varied directly with the size of this correlation.

In Enns and Prinzmetal's (1984) study, the subjects learned of the correlation between the representations of the targets alone and targets in context during the course of the experiment. Many other experiments have dealt with stimuli, such as words or letters, with which the subjects were thoroughly familiar prior to the experiment. Much of this work has been concerned with the wellknown word-superiority effect, which is demonstrated if a target letter is identified more easily in a word than in isolation (Reicher, 1969). If the target letters are presented in a context of other letters that are chosen so that the target letters and context do not form words, then a context-inferiority effect is usually found; that is, the presence of the "noise letters" impairs the subjects' performance unless the distance of the nearest noise letter from the target is quite large (Estes, 1972, 1974; Gardner, 1973; Shiffrin \& Geisler, 1973).

A number of investigators have pointed out the analogy between the word-superiority effect and the effects observed with materials that are not obviously linguistic (Weisstein \& Harris, 1974; Williams \& Weisstein, 1978). However, whether the analogy reflects a similarity in the underlying processes is still under dispute (for a recent detailed discussion of this matter, see Reingold \& Jolicoeur, 1993).

In the experiments reported in this paper, we used pigeons as subjects. Part of the rationale for using pigeons is that comparison of human performance with that of these subjects, whose performance in many visual tasks is similar to that of humans, ${ }^{1}$ may provide evidence that bears directly on theories such as those discussed above. For example, if context-superiority effects are the result of representing the various stimuli in memory by a verbal code, then such context-superiority effects should not be obtained with pigeons. If, contrary to this expectation, such effects were obtained with pigeons, that finding would indicate that there are possible nonlinguistic processes that result in context-superiority effects.

In fact, context-superiority effects have not been obtained with pigeons. Donis and Heinemann (1993) investigated the object-line situation with pigeons as subjects. This situation, which has yielded very robust contextsuperiority effects with human subjects (Enns \& Prinzmetal, 1984; Pomerantz et al., 1977), yielded equally robust context-inferiority effects with pigeons. Context-inferiority effects have also been found with several other stimulus configurations (Donis, 1991; Heinemann \& Chase, 1990), including some in which the stimuli were letters (Steele, 1990).

Most models for the processes that govern the degree of impairment found in the experiments with human subjects ultimately attribute the impairment to confusions between the target and noise letters (Estes, 1972, 1974; Gardner, 1973; Shiffrin \& Geisler, 1973). Some models assume that the probability of such confusions is affected by inhibitory interactions among the inputs to the feature detectors assumed to be involved in perception of the letters; an example is the interactive channels model proposed by Estes $(1972,1974)$. Such a model could account for the context-inferiority effect obtained by Donis and Heinemann (1993), at least in a qualitative way, by assuming that the larger figures (the lines in context) are degraded more than the smailer ones (the targets) because they activate more input channels. Models that do not assume interactions among the input channels (Gardner, 1973; Shiffrin \& Geisler, 1973) do not seem to be directly applicable to the Donis and Heinemann situation, because the experimental procedure for demonstrating an objectline effect in pigeons does not yield any measure of how well a subject detects the presence of a target that is embedded in a context. Instead, it compares the accuracy of discriminating between two targets shown in isolation with the accuracy of discriminating between two more complex forms constructed by embedding each target in an identical context.

We undertook the research described in this paper in the hope of uncovering some of the factors that determine whether the addition of common elements to stimuli results in compounds that are easier or more difficult to discriminate than the stimuli without the common elements.

A further purpose of this research was to test the applicability of a quantitative model of pattern recognition, proposed by Heinemann and Chase (1990), to visual context effects. This model is sufficiently explicit about memory storage, retrieval, and decision processes to make numerical predictions of experimental data. It will be described briefly in the General Discussion section of this paper.

We report three experiments that were conducted in an experimental environment in which contextual stimuli other than those under study were eliminated. In Experiment 1 we explored the role of contextual stimuli as a frame of reference that may help subjects discriminate between targets that differ only in their position in the visual field, as was true in some of the experiments by Williams and Weisstein (1978) and Enns and Prinzmetal (1984). In Experiments 2 and 3 we examined the effects of noninformative contextual stimuli on discrimination between targets that differ in form.

\section{EXPERIMENT 1}

In experimental and theoretical analyses of context effects, the context considered is typically that manipulated by the experimenter. Constant features of the visual environment, such as the edges of the display surface or other landmarks, are not taken into account. What is usually referred to as the "targets-alone" condition is a condition in which the targets are not accompanied by the contexts that the experimenter is interested in, but are accompanied by other visual contexts. It seems to us that if the targets that are studied differ only in their position in the visual field, then what is usually referred to as the 
targets-alone condition may not represent an adequate baseline for assessing the effect of the contexts that the experimenter wishes to study. The principal purpose of Experiment 1 was to assess the effect of the experimentally manipulated contexts against a baseline of a true targets-alone condition.

In an attempt to eliminate unplanned visual cues that might serve as landmarks, the experiment was conducted in a test chamber that was as close to being totally dark as we were able to arrange. In any case, it was dark enough so that no unintended landmarks were visible to a moderately dark-adapted human observer.

The targets used in the experiment were the vertically aligned dots that are numbered Stimulus 1 and Stimulus 2 in Figure 1. The two targets differed only in that one was translated horizontally by $0.5 \mathrm{~cm}$ with respect to the other. This distance represents about $5^{\circ}$ of visual angle if the pigeon views the target from a distance of approximately $5.5 \mathrm{~cm}$ (the F2 position, see Goodale, 1983). In the absence of additional visual stimuli that can serve as a frame of reference, human observers cannot localize such targets accurately, even if the position of their heads is not changed during the interval between the exposure of the two targets (Ludvigh, 1953; Matin, Matin, \& Kibler, 1966). If the same is true of pigeons, then two targets that differ only in retinal location should be indistinguishable when viewed in isolation, but would be expected to be distinguishable when placed in contexts that appear in fixed spatial positions. In the stimuli numbered 3-10 in Figure 1, these targets were accompanied by contexts consisting of pairs of triangles, to be referred to as flankers. The addition of flanking forms at fixed spatial coordinates, as in the stimulus pairs numbered 3 and 4,5 and 6,7 and 8 , and 9 and 10 , produces pairs of compound stimuli that differ geometrically in that the target is somewhat nearer the left flanker than the right one in the odd-numbered stimuli; the reverse is true for the even-numbered stimuli.

\section{Method}

Subjects. The subjects were 2 male and 2 female White Carneaux pigeons, with ages ranging from 2 to 9 years. They had previously served in experiments in which they were trained to discriminate between visual stimuli that were very different from those used in the present experiment. The pigeons were maintained throughout the experiment at approximately $80 \%$ of their mean free-feeding body weights. Between daily experimental sessions, they were housed individually in a colony room where water and grit were constantly available.

Apparatus. All experimental contingencies, as well as the form of the stimuli and the recording of data, were controlled by a Tandy Model 1000 microcomputer with an interface unit (Alpha Products, A-Bus). The subjects were trained and tested in a darkened chamber, the walls of which were painted flat black. The interior dimensions of the chamber were $51 \mathrm{~cm}$ high $\times 57 \mathrm{~cm}$ wide $\times 46 \mathrm{~cm}$ deep. One wall of this chamber had a $23 \mathrm{~cm}$ high $\times 28 \mathrm{~cm}$ wide opening covered by a sheet of transparent plastic, behind which was the viewing screen of a color monitor (Tandy, Model CM-11). A small cup, into which food pellets (Noyes, $45 \mathrm{mg}$, Formula C1) could be automatically delivered, was placed to the left and below the lower edge of the frame. This cup could be illuminated by a small amber light.
During the experiment, a pigeon was required to peck at specific regions on the screen. In order to locate the position of the pigeon's peck, a position-sensing system that was based on a series of infrared emitters and detectors (BFANM Corp., Model 1210) was mounted in front of the monitor. The device consisted of pairs of emitters and detectors that were arranged to form a grid of infrared light beams ( 24 horizontal beams $\times 32$ vertical beams). The grid was scanned every $8 \mathrm{msec}$ to determine the position of any pair of crossed infrared beams interrupted by the pigeon's head. Reward was contingent upon interrupting a designated pair of crossed beams while striking the screen with sufficient force to be detected by two small microphones (Radio Shack, Cat. No. 270$090)$ mounted near the top of the plastic panel. When these conditions were met, the peck was followed by a 20 -msec sound at approximately $2.8 \mathrm{~Hz}$.

Stimuli. The computer monitor was set at low resolution (160 horizontal $\times 200$ vertical pixels). The stimuli were composed of groups of luminous pixels presented on an otherwise dark screen; these closely spaced pixels were arranged to form the patterns shown in Figure 1. Only one of these patterns was shown on any given trial. The odd-numbered stimuli required one response, and the evennumbered stimuli required an alternative response. Each of the targets consisted of two pairs of adjacent pixels, placed one above the other with a $0.5-\mathrm{cm}$ separation between pairs. One of the targets appeared $0.25 \mathrm{~cm}$ to the left of the center of the viewing screen, and the other appeared $0.25 \mathrm{~cm}$ to the right of that center. Each target could be presented alone (the stimuli numbered either 1 or

\section{STIMULI}

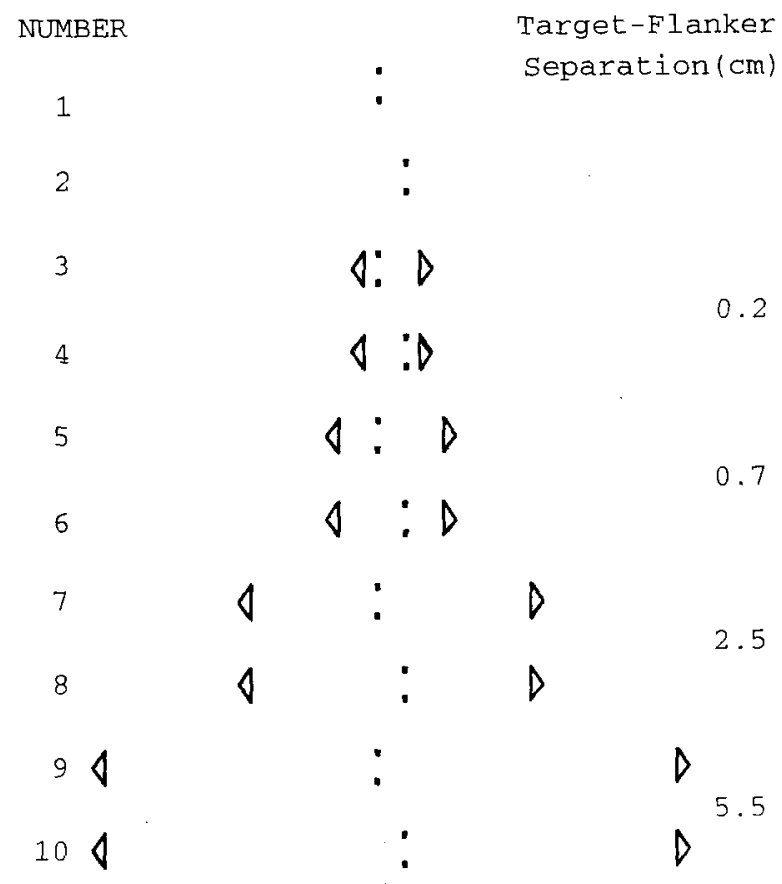

Figure 1. The 10 stimuli used in Experiment 1. These stimuli were white, presented on a completely dark background. Odd-numbered stimuli were positioned so that the target fell $0.25 \mathrm{~cm}$ to the left of the center of the screen, and even-numbered stimuli were positioned so that the target fell $0.25 \mathrm{~cm}$ to the right of that center. The bases of the flanking triangles were equidistant from the center of the viewing screen and were separated from the nearest point on the targets by $0.2,0.7,2.5$, or $5.5 \mathrm{~cm}$. 
2) or accompanied by flankers that consisted of two sets of 18 pixels arranged in the form of triangles. On different trials the flankers were placed at one of four distances (measured from the target to the base of the nearer of the two flankers). The distances were $0.2 \mathrm{~cm}$ for Stimuli 3 and $4,0.7 \mathrm{~cm}$ for Stimuli 5 and $6,2.5 \mathrm{~cm}$ for Stimuli 7 and 8 , and $5.5 \mathrm{~cm}$ for Stimuli 9 and 10 . Because the pair of flankers that accompanied the targets in each of these stimulus pairs was the same, the flankers could not by themselves serve as the basis for discriminating between the members of the pair.

Preliminary training. The subjects were first trained to peck on a white disk $(1.5 \mathrm{~cm}$ in diameter) that appeared in the center of the viewing screen. The size of the disk was gradually reduced over several sessions until it consisted of four adjacent pixels. Pecks on a $1.5 \times 1.5 \mathrm{~cm}$ region surrounding the disk, forceful enough to be sensed by the microphones, produced a brief sound and were followed by delivery of two food pellets.

Discrimination training. Each training session was divided into 100 trials, separated by 9 -sec intertrial intervals (ITIs). The screen appeared white during the ITI and had a luminance of $75 \mathrm{~cd} / \mathrm{m}^{2}$. At the beginning of each trial the screen became dark, and one of the 10 patterns shown in Figure 1 appeared in the center of the screen. More specifically, the center of the screen was located midway between the two flankers. To ensure that the subjects viewed the stimuli, they were required to peck at least once on a $1 \times 1 \mathrm{~cm}$ region in the screen's center. Following this, the stimulus pattern disappeared and was replaced by two light-blue response disks, which were approximately $1.5 \mathrm{~cm}$ in diameter and located to the left and right (with a separation of $6.5 \mathrm{~cm}$ ) and about $1 \mathrm{~cm}$ above the location in which the uppermost part of the stimulus to be identified had been presented. A peck on the left disk was designated a correct response after the target pattern positioned to the left of the center had been presented (any one of the odd-numbered stimuli), and a peck on the right disk was designated a correct response after the target positioned to the right of the center (any one of the even-numbered stimuli) had been presented (whether or not the target was accompanied by flankers).

Correct responses were followed by delivery of two food pellets, as well as illumination of the food cup and the viewing screen for the 9-sec ITI that followed. An incorrect response was followed by a 15-sec ITI in total darkness, and the stimulus incorrectly classified was presented again on the next trial-a correction trial. Correct responses on correction trials were reinforced, but performance on correction trials was excluded from the data analysis. The 10 stimuli were presented in random order with the constraint that each would appear 10 times per session.

During the initial phase of discrimination training, one target pattern was white and the other target pattern was light red, so that both the position and color of the target could serve as cues to the correct response. This phase of training continued for each subject until its performance met a criterion of $80 \%$ correct or better for 2 successive sessions. All the subjects met this criterion within 8 sessions. After the criterion was met, the color difference was eliminated (with all stimuli shown in white). Training was then continued for $\mathbf{5 0}$ sessions, at which point it appeared that none of the subjects were likely to improve with further training.

\section{Results and Discussion}

The results discussed here are those obtained after the color cues had been eliminated. All 4 subjects were trained for 50 sessions, but 1 of them did not acquire the discrimination. The results for this subject were excluded from further analyses. Figure 2 shows the mean learning curves for the 3 subjects that did acquire the discrimination. To avoid overcrowding, only the curves for the target patterns alone and the smallest $(0.2 \mathrm{~cm})$ and largest $(5.5 \mathrm{~cm})$ target-flanker separations are shown.

There was little change in the proportions correct as a function of training when the flankers were either absent or placed at the greatest distance from the target $(5.5 \mathrm{~cm})$. Averaged across the 50 sessions, the proportion correct was .529 for the targets alone and .548 for the compound stimuli with the largest target-flanker separation. Two binomial tests, each based on 1,500 trials,

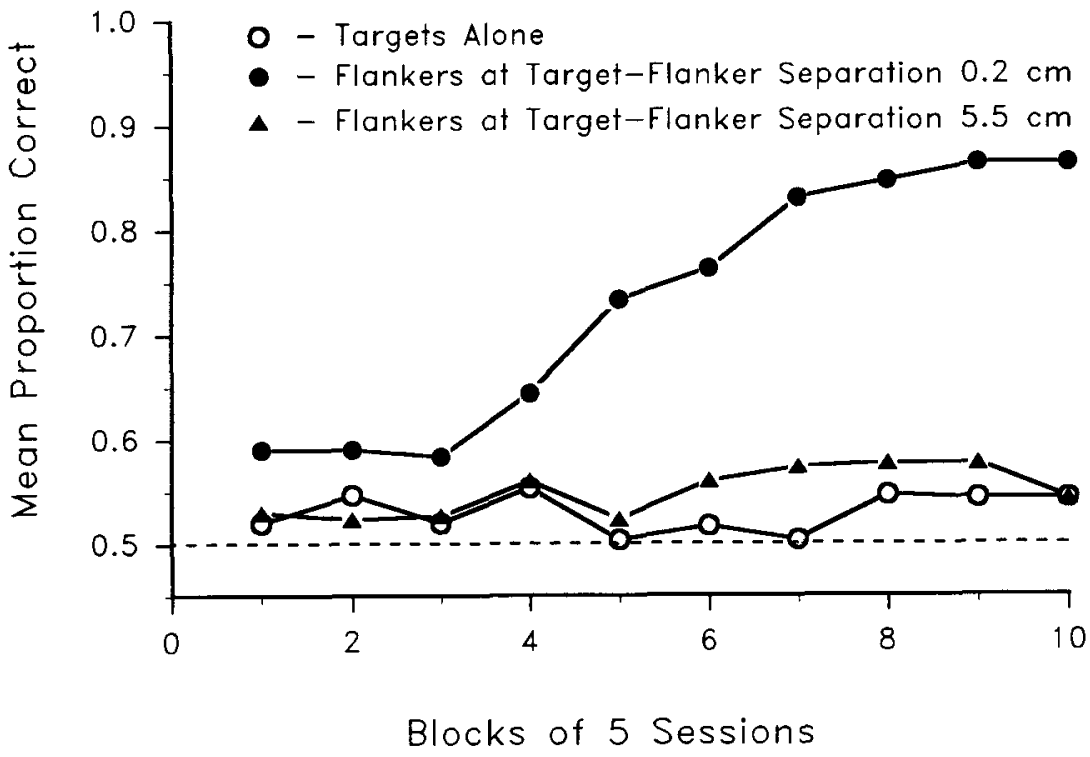

Figure 2. Acquisition of the discrimination in Experiment 1. Mean proportion correct as a function of blocks of training sessions for the 3 subjects that acquired the discrimination. Shown are the learning curves for the targets alone and targets with horizontal flankers at distances of 0.2 or $5.5 \mathrm{~cm}$ from the nearer target. 
showed that each of these two small deviations from the chance level of .50 was significant $(p<.05)$.

The two learning curves under discussion differ sharply from the one obtained with the smallest target-flanker separation. Under the latter condition, learning progressed rapidly and performance became quite accurate.

The filled circles in Figure 3 represent the mean proportions correct during the last 30 sessions of training at each of the four target-flanker separations and in the absence of flankers. The unfilled triangles represent the outcome of a computer simulation based on the HeinemannChase (1990) model, which will be described in the General Discussion section. The target-flanker separations along the horizontal axis are spaced logarithmically. The purpose of this particular spacing was simply to produce a function that is approximately a straight line-a state of affairs that is desirable because our statistical analysis is based on linear regression. The linear relationship between the logarithm of the four separations and the proportion correct that is evident in Figure 3 was confirmed by a regression analysis. For this and subsequent regression analyses, each proportion correct $(p)$ used in the analysis was transformed to the arcsine of $p^{1 / 2}$, a variancestabilizing transformation proposed by Freeman and Tukey (1950) for binomial data. The regression analysis showed the linear relationship to be highly significant $[r(2)=.985, p=.015]$.

The near-chance performance in the absence of flankers that we found in the present experiment suggests that our attempt to eliminate uncontrolled landmarks was reasonably successful.

The context-superiority effect observed in this experiment is not really surprising if one considers the fact that, when the targets are accompanied by flankers, the subject is discriminating between two compound stimuli that differ geometrically. However, the decrease in the accuracy of the discrimination with increasing target-flanker separation requires further explanation. A somewhat superficial level of explanation may be attained by relating it to Weber's law for visual extent, which is known to hold for pigeons, at least under conditions similar to those of the present experiment (Schwabl \& Delius, 1984). The reason we consider this level of explanation superficial is that it does little more than relate one unexplained phenomenon to another unexplained phenomenon.

In any case, the relation between the two phenomena may be clarified by considering the example in which a pigeon is shown one of the four stimuli numbered 3,4 , 9 , or 10 in Figure 1 on each of a series of trials, and is trained to make one response when presented with Stimuli 3 or 9 , and an alternative response when presented with Stimuli 4 or 10 .

If the flankers are the only available landmarks, then the only feature of the visual stimuli that could cue correct responding is that the absolute distance from the target to the left flanker in Stimuli 3 and 9 is slightly smaller than the absolute distance to the right flanker, and the reverse is true in Stimuli 4 and 10. The difference in the

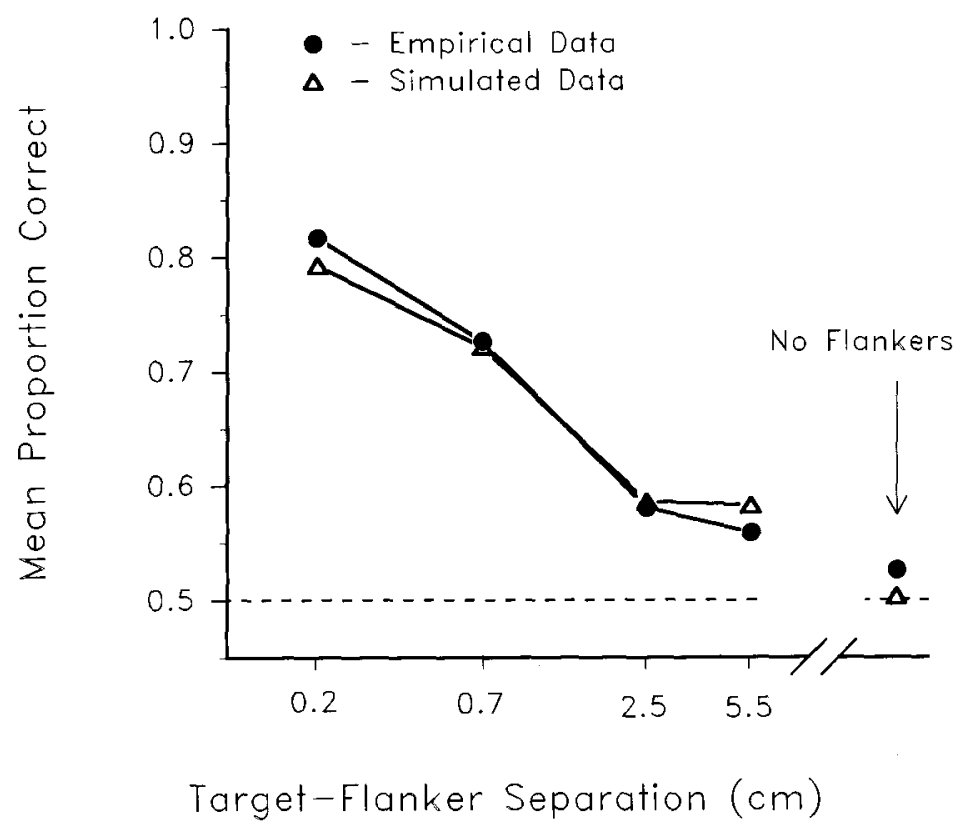

Figure 3. Results of Experiment 1. Mean proportion correct as a function of target-flanker separation, deñned as the distance from the centroid of the target to the base of the nearer of the two flankers. The means (represented by filled circles) are based on results for the 3 subjects that acquired the discrimination and reflect performance during the last 30 training sessions. The unfilled triangles represent the results of a simulation based on the Heinemann-Chase model. The spacing of the target-flanker separations is logarithmic. 
absolute distances of the targets from the left and right flankers is the same for Stimuli 3 and 4, in which the distance to the nearer flanker is $0.2 \mathrm{~cm}$, as it is for Stimuli 9 and 10 , in which the distance to the nearer flanker is $5.5 \mathrm{~cm}$. In relative terms, the difference in the distance to the left and right flankers that must be discriminated for correct responding is very much larger when the stimuli are 3 and 4 (55\% of the distance to the right flanker) than when the stimuli are 9 and 10 (4\% of the distance to the right flanker). Thus, if Weber's law holds, one would expect the discrimination between Stimuli 3 and 4 to be much better than that between Stimuli 9 and 10 .

\section{EXPERIMENT 2}

Experiment 1 showed that, if presented in isolation, targets that differ only in spatial location were discriminated from each other at a level that barely (though significantly) exceeded the chance level of $50 \%$ correct. Embedding such targets in a context of redundant stimuli that can serve as landmarks makes a substantial discrimination between the resulting targets in context possible. The contexts we presented (the flankers) were uninformative in the sense that in isolation they could not be used to identify the correct responses. On that basis, the effect that was found could be classified as a context-superiority effect.

In contrast to the effect found in Experiment 1, several pigeon experiments cited in the introduction showed that discrimination between targets that differ in shape are harmed rather than helped by the addition of noninformative contexts. There is no reason to think that the difference between the findings of these experiments and those of the present Experiment 1 is attributable to the pseudoGanzfeld conditions of the latter. The theoretical accounts of context effects discussed in the introduction, as well as the account given by the Heinemann-Chase (1990) model, propose that embedding targets in contexts generally leads to context-inferiority effects. It seems reasonable to assume that the processes believed to play a role in producing the context-inferiority effect operate under the conditions of Experiment 1 also, but that their influence is outweighed by the positive effects of the frameof-reference function of the contexts. If that is so, then contexts can be expected to have a helpful effect, such as that found in Experiment 1, only if a discrimination between the targets is either impossible without these contexts or can be considerably improved by their presence. The main purpose of Experiment 2 was to find out whether context-inferiority effects would replace the context-superiority effects under our viewing conditions, if targets that did not require a visual frame of reference to be discriminated from each other were used. To this end, two new targets were created by accompanying each of the targets used in Experiment 1 with a pair of nearby landmarks.

\section{Method}

Subjects. The subjects were the 3 pigeons that had learned the discrimination in Experiment 1. They were maintained as in Experiment 1 .
Apparatus and Stimuli. The apparatus was the same as that used in Experiment 1. The two targets were the two sets of vertically aligned pixels, each placed between the nearest horizontal flankers (Figure 1, Stimuli 3 and 4). Each of these redefined targets was presented alone or in combination with two vertical flankers that were placed above and below the targets. To distinguish between the two sets of flankers, the new ones will be called vertical flankers and those also used in Experiment 1 will be called horizontal flankers. The bases of the vertical flankers were separated from the nearest point on the targets by the same four distances used for the horizontal flankers in Experiment 1 (i.e., 0.2, 0.7, 2.5, and $5.5 \mathrm{~cm}$ ). Thus, as in Experiment 1, 10 stimuli were used. Figure 4 shows 6 of these stimuli; illustrations of the 4 stimuli that involve the two largest (vertical) target-flanker separations were omitted to conserve space.

Procedure. Experiment 2 began immediately after the conclusion of Experiment 1. There was no initial color-discrimination training. The procedure was the same as that used in Experiment 1, except that the subjects were trained for only 30 sessions. Each training session consisted of 100 trials, with the 10 stimuli presented 10 times each in a random order.

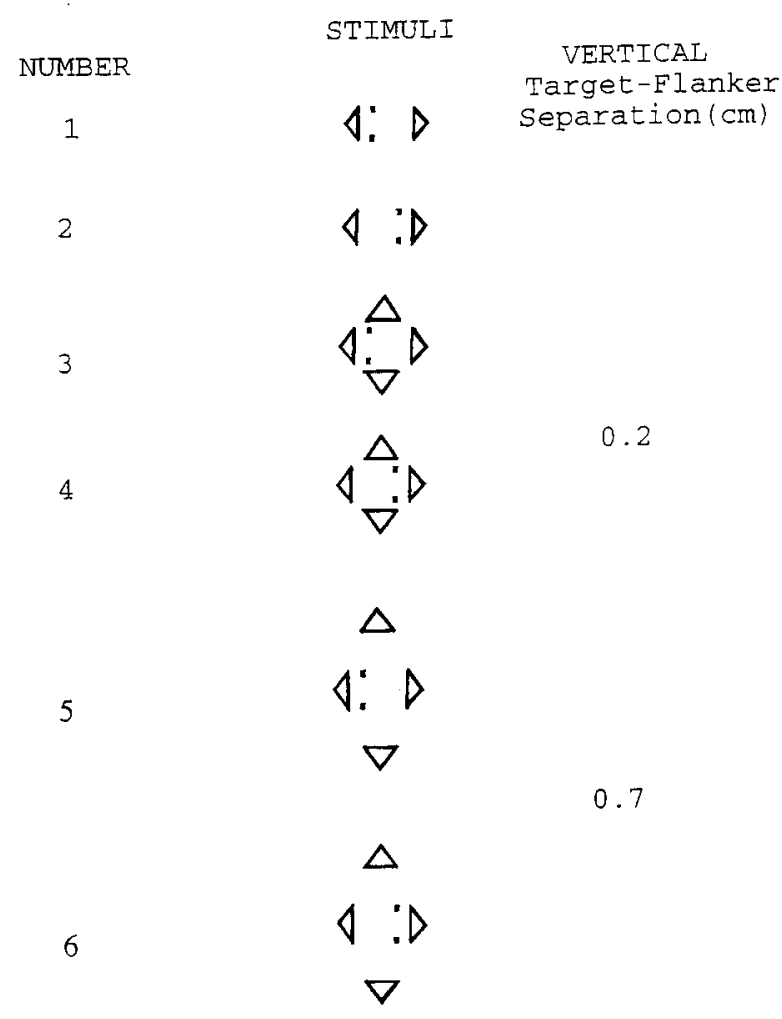

Figure 4. Six of the 10 stimuli used in Experiment 2. These stimuli were white, presented on a completely dark background. The term "vertical target-flanker separation" refers to the shortest distance between one of the vertically aligned points to the base of the nearer of the vertical flankers. All stimulus patterns included horizontal flankers with bases located $0.45 \mathrm{~cm}$ to the left and right of the center of the monitor. Odd-numbered stimuli were positioned so that the vertically aligned points that had served as targets in Experiment 1 fell $0.25 \mathrm{~cm}$ to the left of the center of the screen, and even-numbered stimuli were positioned so that these pixels fell $0.25 \mathrm{~cm}$ to the right of that center. To conserve space, two additional pairs of stimuli used in the experiment are not shown. These differed from the stimuli shown in the figure only in that the vertical target-flanker separations were larger: $2.5 \mathrm{~cm}$ for one pair and $5.5 \mathrm{~cm}$ for the other. 


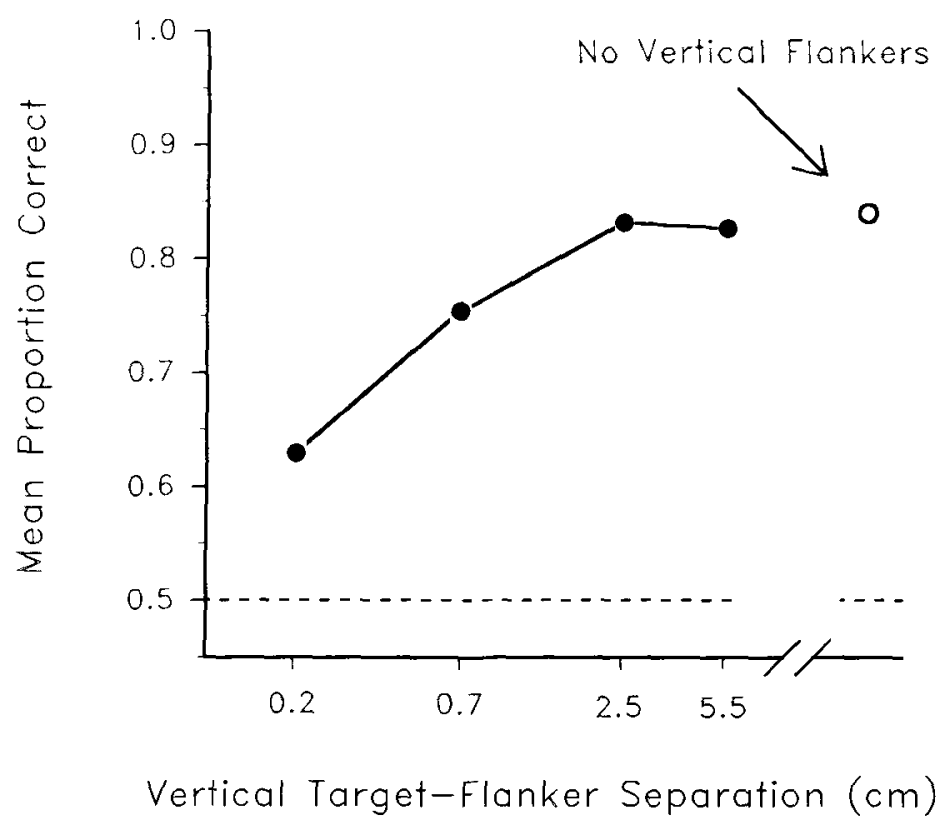

\begin{abstract}
Figure 5. Results of Experiment 2. The filled circles represent the mean proportion correct as a function of the distance of each target from the vertical flankers. The bases of the vertical flankers were separated from the nearest point on the targets by $0.2,0.7,2.5$, or $5.5 \mathrm{~cm}$. All stimuhus patterns included horizontal flankers ("landmarks"), with bases located $0.45 \mathrm{~cm}$ to the left and right of the center of the monitor. The unfilled circle represents the result obtained when the vertical flankers were omitted. The means are based on results of $\mathbf{3 0}$ training sessions.
\end{abstract}

\section{Results and Discussion}

Figure 5 shows the mean proportions correct for the 3 subjects as a function of vertical target-flanker separation. The unfilled circle represents responding in the absence of the vertical flankers. The proportion correct obtained under this condition is quite comparable to that observed under the same condition in Experiment 1. The filled circles show what happened when vertical flankers were also present at one of four distances from the target. The presence of the vertical flankers resulted in a clear context-inferiority effect. The magnitude of this context-inferiority effect decreased with increasing targetflanker separation. At target-flanker separations of 2.5 and $5.5 \mathrm{~cm}$, the accuracy approached that obtained without the vertical flankers.

The results obtained during the 30 days of training for each of the five pairs of stimuli were combined for statistical analysis. The regression analysis showed the linear relationship between proportion correct and the logarithm of the four target-flanker separations to be significant $[r(2)=.954, p=.046]$.

A conceivable explanation for the form of the functions shown in Figure 5 is that the introduction of the vertical flankers disrupted performance at the beginning of the experiment, and that this disruption was most severe for the nearby flankers. The possibility that the proportion of correct responses changed systematically during the $30 \mathrm{ex}-$ perimental sessions was evaluated by a two-way analysis of variance that compared performance during the first five and last five sessions (training blocks), for each of the five stimulus pairs defined by the absence of flankers or one of the four target-flanker separations. The proportions correct for the first and last five sessions did not differ significantly $[F(1,2)=0.77, p>.05]$, and there was no significant interaction of stimulus pair and training block $[F(4,8)=1.02, p>.05]$. However, the effect of stimulus pair was significant $[F(4,8)=12.33, p<$ $.01]$, confirming the outcome of the regression analysis.

\section{EXPERIMENT 3}

In order to obtain some information about the generality of the distractor effect found in Experiment 2, Experiment 3 was done with targets that differed from the ones used in Experiment 1 in that the two points that make up each target were aligned diagonally rather than vertically.

The stimuli used are shown in Figure 6. The target shown in the odd-numbered stimuli was constructed by pairing the top point of the left target used in Experiment 1 with the bottom point of the right target used in that experiment. The target shown in the even-numbered stimuli was constructed by pairing the top point of the right target used in Experiment 1 with the bottom point of the left target used in that experiment. Because the targets 


\section{STIMULI}

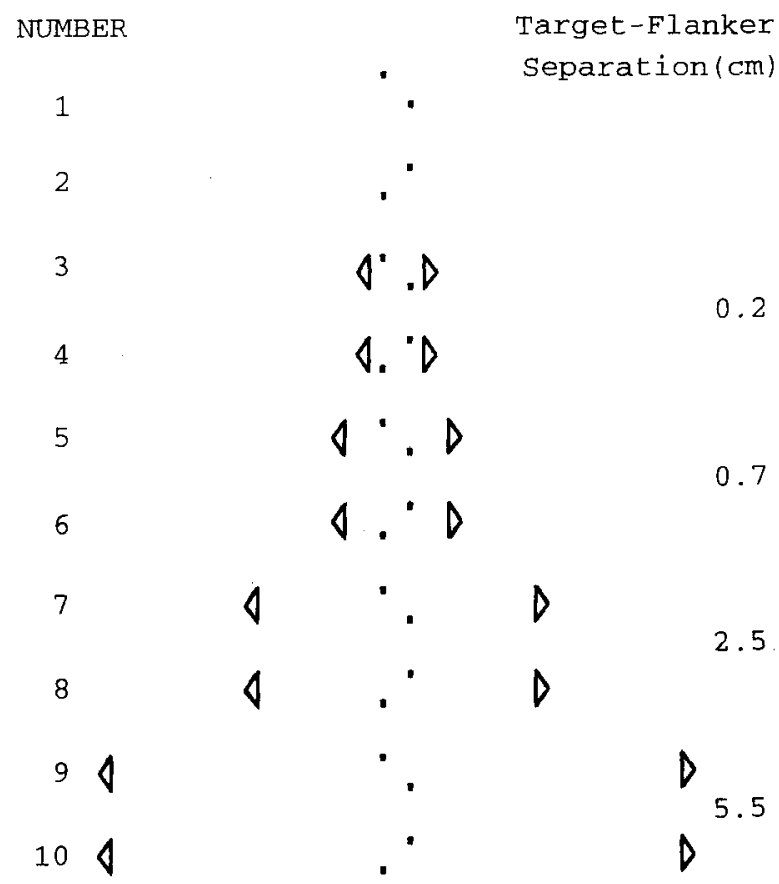

Figure 6. The 10 stimuli used in Experiment 3. These stimuli were white, presented on a completely dark beckground. The targetflanker separation is the distance from each of the two dingonally aligned points that constitute a target to the nearest flanker.

created in this fashion differ in form, we assumed that the pigeons would readily discriminate between them if they were presented one at a time, unaccompanied by any visual frame of reference. If our assumption is correct, then placing each target between two nearby flankers should result in a pair of compound stimuli that are less readily discriminated than the targets alone.

\section{Method}

Subjects. The subjects were 2 male and 2 female White Carneaux pigeons that had not participated in the previous experiments. Their ages ranged between 4 and 8 years. They were maintained as were the pigeons in the previous experiments.

Apparatus. The subjects were trained and tested in an experimental chamber that was similar to the one used in Experiments 1 and 2. The position-sensing system used in Experiments 1 and 2 was replaced by a pressure-sensitive touch screen (Elographics, Model E270) mounted on a convex glass surface in front of the computer monitor. A thin, clear plastic sheet was placed over the touch screen to prevent damage from the pigeons' pecks. A brief sound $(2.8 \mathrm{~Hz})$ could be presented following a peck on a designated region of the screen. Two small cups, into which food pellets could be delivered by automatic dispensers, were mounted below the monitor screenone cup to the left and the other to the right of the screen. Food was delivered to the cup located on the same side as the response disk that was defined as correct in the presence of a given target. A small amber light, mounted above each food cup, could be turned on when food pellets were delivered.
Stimuli and Procedure. The two targets were the stimuli numbered 1 and 2, respectively, in Figure 6. The flankers and target-flanker separations were the same ones used in Experiment 1. At any given target-flanker separation, the centroids of the targets were equidistant from the bases of the flanking triangles. The procedure was the same as that used for Experiment 1. As in that experiment, there was an initial phase of discrimination training during which the two targets differed in color. The color difference was eliminated for each subject after its performance exceeded $80 \%$ correct for 2 successive sessions. Training was then continued for 50 additional sessions.

\section{Results and Discussion}

The 4 subjects were trained for 50 sessions, but only 3 learned the discrimination. The results for the nonlearner were excluded from the analyses described in this section. Figure 7 shows mean learning curves based on the results of the 3 subjects that acquired the discrimination. To avoid overcrowding, the only learning curves that are shown are those for the conditions in which the target patterns were presented alone or were accompanied by flankers at target-flanker separations 0.2 or $5.5 \mathrm{~cm}$.

Because the performance of each of the subjects appeared to have reached a reasonably steady level by the last 30 days of training, the results for these sessions were combined for further analysis. Figure 8 shows the mean proportions correct as a function of target-flanker separation. Not unexpectedly, the results show a clear contextinferiority effect that diminishes with increasing targetflanker separation. The regression analysis showed the linear relationship between proportion correct and the logarithm of the four target-flanker separations to be significant $[r(2)=.964, p=.036]$.

The essential difference between the present experiment and Experiment 1 is as follows. In Experiment 1, the targets alone did not differ geometrically, unless their absolute position in visual space could be determined. However, the compound figures created by combining each target with flankers in identical positions differed intrinsically-in one of the figures the target was positioned nearer to the left flanker than the right one, whereas the reverse was true for the other figure.

In contrast to this, the targets used in the present experiment, when not accompanied by flankers, can be distinguished without knowing their absolute position in space. Adding fixed flankers to these targets does not create geometric differences, as was the case in Experiment 1.

\section{GENERAL DISCUSSION}

The outcomes of our experiments suggest that the addition of noninformative contextual stimuli to targets that pigeons can tell apart in the absence of any added visual context produces compound stimuli that are more difficult to discriminate than the targets without the added contexts. On the other hand, the addition of the same contextual stimuli to targets that differ only in location and cannot be identified without a visual frame of reference improves performance. The size of both of these effects 


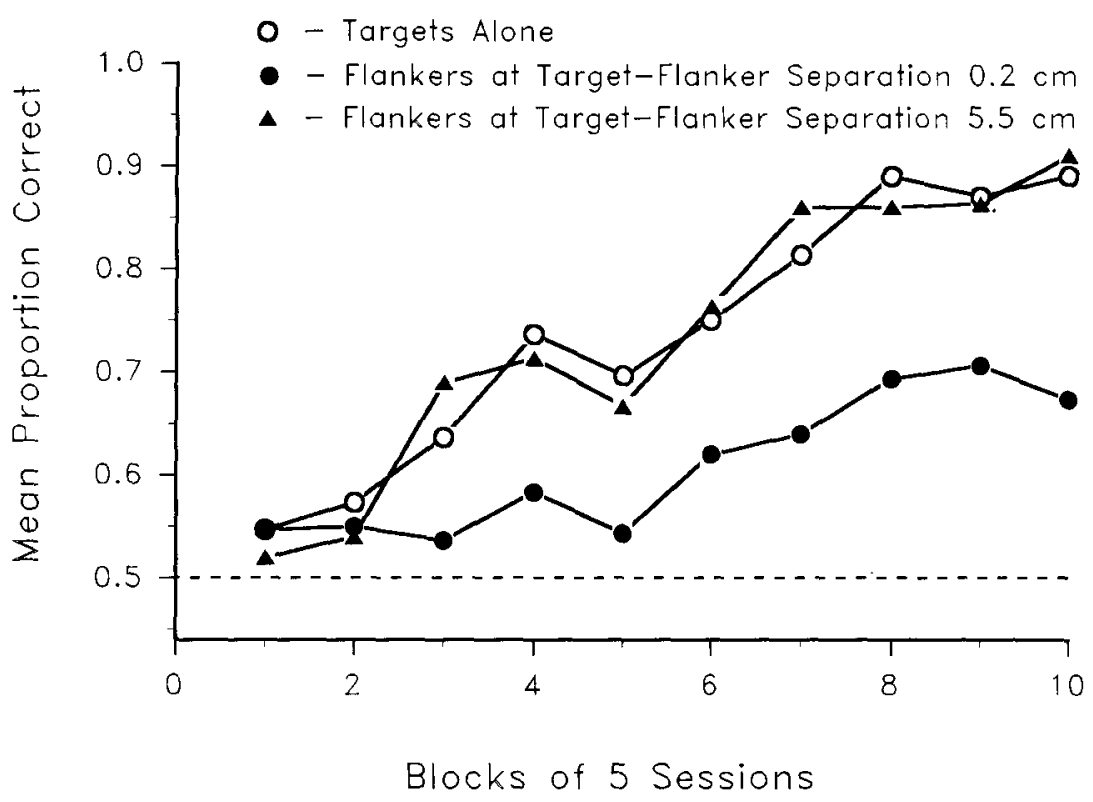

Figure 7. Acquisition of the discrimination in Experiment 3. Mean proportion correct for the 3 subjects that acquired the discrimination, as a function of blocks of training sessions. Shown are the learning curves for the targets alone and targets with horizontal fiankers at distances of 0.2 and $5.5 \mathrm{~cm}$ from the nearer target.

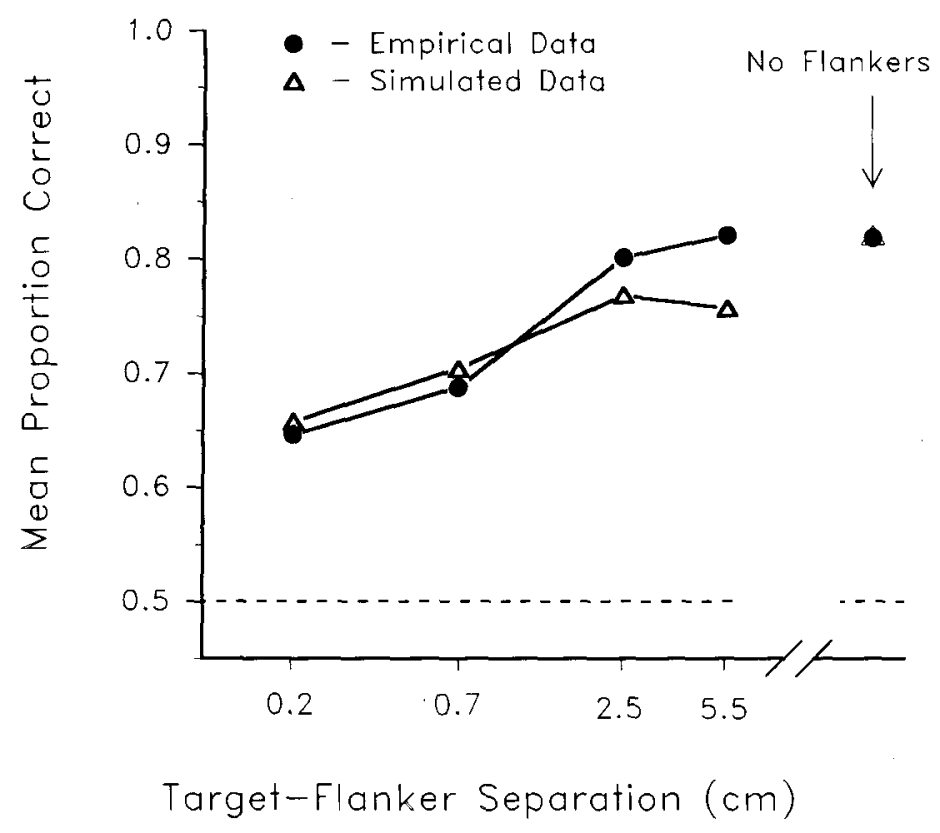

Figure 8. Results of Experiment 3. Mean proportion correct as a function of target-flanker separation. Means are based on results for the 3 subjects that acquired the discrimination and reflect performance during the last $\mathbf{3 0}$ training sessions (filled circles). The unfilled triangles represent the result of a simulation based on the Heinemann-Chase model. The spacing of the targetflanker separations is logarithmic. 
diminishes as the spatial separation between the target and the contextual stimulus elements increases.

\section{The Heinemann-Chase Model}

We wish to briefly consider the interpretation of these results in terms of the Heinemann-Chase (1990) model for visual pattern recognition. This model is part of a much more general model, which is applicable to phenomena that range from discrimination learning and generalization (Heinemann, 1983a, 1983b, 1984) to absolute identification of unidimensional and multidimensional stimuli and the factors that limit the accuracy of such identifications (Chase, 1983; Chase \& Heinemann, 1989, 1991; see Sperling, 1988, for a discussion of the model's application to absolute identification by human subjects).

The present version of this model (Heinemann \& Chase, 1990 ) does not include a treatment of early visual processes that result in interactions among input channels. It is assumed that the effects of such interactions are smaller than the effects of memory and decision processes, at least for the outline forms used in the experiments considered in this paper. For purposes of analysis, the continuum of sensations induced by ongoing visual stimulation is divided into small cells referred to as pixels-a term that usually refers to spatial subdivisions of the distal stimulus. Each pixel is characterized by its spatial coordinates and by a uniform hue, saturation, and brightness. In the experiments considered in this paper, the stimuli were composed of small, rectangular "white" areas of uniform luminance on an otherwise dark computer monitor. Because these areas did not differ from each other in the spectral composition of the emitted light, it is sufficient to specify the pixels by their spatial coordinates.

After training, the subject's long-term memory (LTM) is assumed to contain multiple records of events that occurred on individual trials. Each record shows the sensation experienced during the trial, the response made, and the outcome (reward or nonreward). Because pigeons view stimuli from different distances on different trials before pecking at the stimulus with eyes closed (see Goodale, 1983), it is assumed that the stored representations of the images induced by any given stimulus have a variety of different sizes. It is further assumed that independent Gaussian noise is added to the coordinates of all individual pixels while they reside in LTM.

To select the response to be made on a given trial, the current input, defined as the complex of sensations induced by the current visual stimulation, is compared with that represented on each of a small number of records randomly retrieved from LTM. The only records that are used in the comparison process are those that show a response that was followed by reward. The objective of the comparisons is to find the remembered image that is most similar to the current input, and then to make the response associated with that image.

The comparison of the current input to a remembered input involves superimposing the centroids of these two inputs. The decision quantity (DQ), the index of similar- ity on which the response selection is based, considers the relation of each pixel of the current input to each pixel of the remembered form. Specifically, each pixel of the current input is compared with each pixel of the remembered form in order to calculate, at the location of each input pixel, the mean of the probability densities contributed by the bivariate Gaussian distributions that represent each pixel of the remembered form. This process yields a number of means equal to the number of pixels on the current input. The DQ is the product of these means. The current input is compared with the remembered input shown on each of the records retrieved from LTM in the same way. DQs derived from records associated with the same response label are summed, and the decision rule is: Make the response that yields the largest summed DQ.

Records that represent extreme mismatches to the current input are discarded. In the event that all records in the sample are discarded, a new sample is drawn. If repeated resampling from LTM fails to yield the information needed to decide which response is more likely to lead to reward in the presence of the current input, the subject "guesses." Specifically, the subject selects the response that, according to the records in the current sample, is associated with the larger total reward. The frequency and amount of reward associated with the two responses were equal in the present experiments, so the expected probability of selecting the correct response when guessing occurs is .5 .

\section{Interpretation of the Context-Superiority Effect: Experiment 1}

In interpreting the context-superiority effect found in Experiment 1, we first note that the two targets alone should be indistinguishable because, in the absence of some visual marker, the memories carrying one or the other of the two response labels are indistinguishable. On the other hand, if each target is accompanied by a pair of flankers, the resulting compound stimuli differ geometrically, as noted in the discussion of Experiment 1. Such compound stimuli are discriminable.

We next consider the way that the Heinemann-Chase (1990) model interprets the finding that the helpful effect of the flankers diminished with increasing target-flanker separation in Experiment 1 . In its final form, the interpretation offered is that accuracy decreases with increasing target-flanker separation because the subject guesses more often as the target-flanker separation increases. The mechanism that brings about this effect is dependent on the circumstance, mentioned previously, that pigeons view the stimuli from different distances on different trials. As a result, the retinal image of the object viewed varies in size from trial to trial. Given the assumptions of the model, the trial-to-trial variations in the size of the retinal image have the consequence that a fixed-size object that has been presented on many trials will be represented in memory by a distribution of remembered sizes, rather than by just one size. A given change in viewing distance 
will cause the retinal image of any object in the frontoparallel plane to change its size (distance between any two points on the object) by a constant proportion that is independent of the size of the (distal) object. If the distribution of viewing distances over trials is reasonably symmetric, then the distribution of remembered sizes corresponding to a fixed distal size will have a standard deviation that is proportional to the mean.

The probability that a subject will guess is assumed to increase with the probability that records retrieved from LTM have to be discarded because they contain no useful information-that is, represent severe mismatches. In the situations under consideration, the most frequent reason for mismatches is that records of remembered inputs represent flankers at positions that differ substantially from those of the flankers present in the current input. As mentioned above, trial-to-trial variations in viewing distance will cause the memory representation of the distance between flankers to vary over a larger range if the interflanker distance in the distal stimulus was large rather than if it was small. As a result, the probability that a record will be rejected increases with increasing interflanker distance in either the current input or the record-with attendant increases in the probability of guessing.

The theoretical curve shown in Figure 2 represents the outcome of a computer simulation based on the HeinemannChase (1990) model. It is not a best fit, but a rough approximation based on trial and error procedures during which three parameters were manipulated. One was a measure of the trial-to-trial variations in the pigeons' observation distance, the second determined the number of times the subject was assumed to retrieve a new sample before resorting to guessing, and the third was the distance between neighboring pixels in the internal representation.

It should be noted that the above account does not invoke Weber's law to explain the effects shown in Figure 2. To the contrary, Weber's law for visual extent can itself be derived from the considerations presented in the preceding paragraphs, but this matter is not sufficiently relevant to this paper to justify extensive discussion.

\section{Interpretation of the Context-Inferiority Effect: Experiments 2 and 3}

We turn now to context-inferiority effects, such as those found in Experiments 2 and 3. According to the Heinemann-Chase (1990) model, these arise from the manner in which the DQs for the correct and incorrect responses are obtained. Compared with the DQs expected when current and remembered inputs that represent targets alone are being compared, the DQs expected when the current and remembered inputs representing targets accompanied by flankers are compared are reduced, as is the difference between them. In general, the more pixels that the current input and the remembered pattern have in common, the smaller the difference in the DQs associated with the correct and incorrect responses.
Finally, for the stimulus configurations used in our experiments, the context-inferiority effect is expected to be strongest when the flankers are nearest to the targets. The reason is that, because of the geometry, the effect of flankers placed very near the targets is to increase the $D Q$ associated with the incorrect response much more than that associated with the correct one.

The outcome of a simulation based on this model is shown in Figure 8. The parameters that were varied to obtain this curve are those mentioned in connection with the theoretical curve shown in Figure 3.

\section{Possible Application to Some Effects Found with Human Subjects}

The theoretical account of the particular contextsuperiority effect discussed above might possibly be relevant to the interpretation of some context-superiority effects reported for human observers. For example, Enns and Prinzmetal (1984, Experiment 1) used stimulus displays in which the two target lines differed only in their position in the visual field. All of the subjects served in two experimental sessions. In the first session, the target lines were presented alone on half of the trials. On the remaining trials, they formed part of a larger figure - the context. Ten different contexts were studied, but each individual subject was exposed to only one of these contexts during the first session. This procedure yielded strong context-superiority effects for all 10 contexts.

The human observers in Enns and Prinzmetal's (1984) experiment were able to distinguish the positions in which the lines appeared, even in the absence of the experimentally manipulated context. There is no reason to think that they would be able to do this if the lines were presented one at a time in a Ganzfeld, as we attempted to do in the experiments reported here. Presumably, the subjects were able to distinguish the positions in which the lines appeared by using distant contextual stimuli, such as the edges of the display surface or perhaps more subtle contextual stimuli, as fixed landmarks. The experimentally manipulated contexts might well have caused the observed improvement in identification performance simply by providing landmarks that were closer to the target lines.

During the second session, Enns and Prinzmetal (1984) presented their subjects with each of the single lines, and with each of these lines embedded in 10 different contexts (a total of 22 different stimuli). Each subject was exposed to all 22 of these stimuli during the second experimental session. This differed from the procedure used during the first session, in which each subject was exposed to only four stimuli-each of the single lines and each of these lines embedded in an identical context. Each of the 10 contexts used in the second session was presented to a different group of subjects in the first session. In both of the experimental sessions the single lines were shown on half of the trials, and the lines in context were shown on the remaining trials. Under these conditions, all of the lines in context yielded significant context- 
superiority effects in the first session, whereas significant context-inferiority effects were found in the second session.

A comparable experiment has not been done with pigeons. However, the assumptions concerning memory storage and retrieval processes that are at the basis of the Heinemann-Chase (1990) model lead to predicted outcomes that are qualitatively similar to those reported by Enns and Prinzmetal (1984). To gain an intuitive understanding of the causes of the context-inferiority effect observed in Enns and Prinzmetal's experiment, it is useful to consider the composition of a sample of records randomly retrieved from LTM during Session 2 of that experiment. On the average, half of the records in the sample will represent one or the other of the single lines, and each of the remaining records will represent 1 of the 20 lines in context. However, because the number of records retrieved in a single sample is assumed to be quite small, records that represent any one of the 20 lines in context that may appear as the current input will often fail to appear in that sample. Thus, any particular sample is more likely to contain a record that represents a match to one of the single lines than one that represents a match to one of the lines in context.

The fact that many of the lines in context are quite similar to each other would be expected to lead to some incorrect response choices rather than to rejection of nonmatching records. These factors would cause the error rate for lines in context to be greater than it was in the first session, which is what was found. Though stated in very different terms, the possibility that the poor recognition of the lines in context during the second session might reflect memory failure was also suggested by Enns and Prinzmetal (1984).

Though there may be some conditions under which the effects of contextual stimuli on recognition are similar in humans and pigeons, perhaps because of similar underlying processes, the much-studied configuration in which two diagonal lines are either shown alone or embedded in identical L-shaped contexts (e.g., Donis \& Heinemann, 1993; Enns \& Prinzmetal, 1984, Experiment 2; Pomerantz et al., 1977) produces vastly different results for pigeons and humans. As mentioned in the introduction, it produces robust context-superiority effects in humans, but equally robust context-inferiority effects in pigeons. It seems likely that the context-superiority effect observed with human subjects in this situation reflects a level of processing not found in pigeons-perhaps a redundancy gain due to the creation of a dimension such as "trianglearrow" that is correlated with the angular orientation dimension of the single lines, as proposed by Enns and Prinzmetal.

\section{REFERENCES}

BLough, D. S. (1985). Discrimination of letters and random dot patterns by pigeons and humans. Journal of Experimental Psychology: Animal Behavior Processes, 11, 261-280.

Chase, S. (1983). Pigeons and the magical number seven. In M. L.
Commons, R. J. Herrnstein, \& A. R. Wagner (Eds.), Quantitative analyses of behavior: Discrimination processes (Vol, 4, pp. 37-57). Cambridge, MA: Ballinger.

Chase, S., \& Heinemann, E. G. (1989). Effects of stimulus complexity on identification and categorization. Intermational Joumal of Comparative Psychology, 3, 165-181.

Chase, S., \& Heinemann, E. G. (1991). Memory limitations in human and animal signal detection. In M. L. Commons, J. A. Nevin, \& M. C. Davison (Eds.), Signal detection: Mechanisms, models, and applications (pp. 121-137). Hillsdale, NJ: Erlbaum.

DoNIS, F. D. (1991). The effects of non-informative stimulus elements on pattern recognition by pigeons. Dissertation Abstracts International, 52 (05B). (University Microfilms No. 91-30, 312)

Donis, F. D., \& Heinemann, E. G. (1993). The object-line inferiority effect in pigeons. Perception \& Psychophysics, 53, 117-122.

Enns, J. T., \& Prinzmetal, W. (1984). The role of redundancy in the object-line effect. Perception \& Psychophysics, 35, 22-32.

EsTES, W. K. (1972). Interactions of signal and background variables in visual processing. Perception \& Psychophysics, 12, 278-286.

EsTEs, W. K. (1974). Redundancy of noise elements and signals in the visual detection of letters. Perception \& Psychophysics, 16, 53-60.

Freeman, M. F., \& TukeY, J. W. (1950). Transformations related to the angular and the square root. Annals of Mathematical Statistics, 21, 607-611.

Fujrta, K., Blough, D. S., \& Blough, P. M. (1991). Pigeons see the Ponzo illusion. Animal Leaming \& Behavior, 19, 283-293.

Fujita, K., Blough, D. S., \& Blough, P. M. (1993). Effects of the inclination of context lines on perception of the Ponzo illusion by pigeons. Animal Learning \& Behavior, 21, 29-34.

GARDNER, G. T. (1973). Evidence for independent parallel channels in tachistoscopic perception. Cognitive Psychology, 4, 130-155.

GoOdALE, M. A. (1983). Visually guided pecking in the pigeon (Columba livia). Brain, Behavior \& Evolution, 22, $22-41$.

HeInEManN, E. G. (1983a). A memory model for decision processes in pigeons. In M. L. Commons, R. J. Herrnstein, \& A. R. Wagner (Eds.), Quantitative analyses of behavior: Discrimination processes (Vol. 4, pp. 3-19). Cambridge, MA: Ballinger.

HeinemanN, E. G. (1983b). The presolution period and the detection of statistical associations. In M. L. Commons, R. J. Herrnstein, \& A. R. Wagner (Eds.), Quantitative analyses of behavior: Discrimination processes (Vol. 4, pp. 21-35). Cambridge, MA: Ballinger.

HeinemanN, E. G. (1984). A model for temporal generalization and discrimination. In J. Gibbon \& L. Allan (Eds.), Timing and time perception (Annals of the New York Academy of Sciences, Vol. 423, pp. 361-371). New York: New York Academy of Sciences.

HeinemanN, E. G., CHASE, S. (1990). A quantitative model for pattern recognition. In M. L. Commons, R. J. Herrnstein, S. M. Kosslyn, \& D. B. Mumford (Eds.), Quantitative analyses of behavior: Computational and clinical approaches to pattem recognition and concept formation (Vol. 9, pp. 109-126). Hillsdale, NJ: Erlbaum.

Herrnstein, R. J. (1979). Acquisition, generalization, and discrimination reversal of a natural concept. Journal of Experimental Psychology: Animal Behavior Processes, 5, 118-129.

LuDVIGH, E. (1953). Direction sense of the eye. American Journal of Ophthalmology, 36, 139-153.

Mallot, R. W., \& MaLlot, M. K. (1967). The effect of outward pointing arrowheads on the Mueller-Lyer illusion. Psychonomic Science, 9, 55-56.

Mallot, R. W., \& StdDal, J. W. (1972). Acquisition of the people concept in pigeons. Psychological Reports, 31, 3-13.

Matin, L., Matin, E., \& KIBLER, G. (1966). Visual perception of direction in the dark: Roles of local sign, eye movements, and ocular proprioception. Vision Research, 6, 453-469.

Pomerantz, J. R., Sager, L. C., \& Stoever, R. J. (1977). Perception of wholes and their component parts: Some configural superiority effects. Joumal of Experimental Psychology: Human Perception \& Performance, 3, 422-435.

REICHER, G. M. (1969). Perceptual recognition as a function of the meaningfulness of the material. Journal of Experimental Psychology, 81, 275-280.

Reingold, E. M., \& Joucoeur, P. (1993). Perceptual versus postper- 
ceptual mediation of visual context effects: Evidence from the lettersuperiority effect. Perception \& Psychophysics, 53, 166-178.

Schwabl, U., \& DeluUs, J. D. (1984). Visual bar length discrimination threshold in the pigeon. Bird Behavior, 5, 118-121.

Shiffrin, R. M., GeIsLeR, W. S. (1973). Visual recognition in a theory of information processing. In R. L. Solso (Ed.), Contemporary issues in cognitive psychology: The Loyola symposium (pp. 53-101). New York; Halsted Press.

SPERLING, G, (1988). The magical number seven: Information processing then and now. In W. Hirst (Ed.), The making of cognitive science: Essays in honor of George A. Miller (pp. 71-80). Cambridge: Cambridge University Press.

STEELE, R. (1990). Configural processes in pigeon perception. In M. L. Commons, R. J. Herrnstein, S. M. Kosslyn, \& D. B. Mumford (Eds.), Quantitative analyses of behavior: Behavioral approaches to pattern recognition and concept formation (Vol. 8, pp. 111-125), Hillsdale, NJ: Erlbaum.

WeISSTEIN, N., \& HaRRIS, C. (1974). Visual detection of line segments: An object superiority effect. Science, 186, 752-755.
Williams, A., \& Weisstein, N. (1978). Line segments are perceived better in a coherent context than aione: An object-line effect in visual perception. Memory \& Cognition, 6, 85-90.

\section{NOTE}

1. For example, pigeons and humans make similar errors in identifying letters of the alphabet (D. S. Blough, 1985), and pigeons are subject to visual illusions similar to those experienced by humans $(R$. W. Mallot \& M. K. Mallot, 1967; Fujita, D. S. Blough, \& P. M. Blough, 1991, 1993). Pigeons have also been trained to categorize photographs of natural scenes on the basis of buman concepts, such as the presence or absence of trees, water, or human figures; after training they are able to categorize new instances of the concept at better than chance levels (Herrnstein, 1979; R. W. Mallot \& Siddal, 1972).

(Manuscript received January 29, 1993; revision accepted for publication November 1, 1993.) 